\title{
Correlation Between Infrared Intensities, Dissociation Energies, and Equilibrium Internuclear Distances for Diatomic Molecules*
}

\author{
HENRY AROESTE \\ Guggenheim Jet Propulsion Center, California Institute of Technology \\ Pasadena, Californi
}

(Received April 19, 1954)

$\mathrm{O}^{\mathrm{n}}$ NE may expand the dipole moment as a function of internuclear distance as follows:

$$
\mu=\mu_{0}+\left(\frac{\partial \mu}{\partial r}\right)_{r=r_{0}}\left(r-r_{0}\right)+\cdots .
$$

The derivative $(\partial \mu / \partial r) r=r_{0}$ may be obtained from infrared intensity measurements. Using the best available data of $(\partial \mu / \partial r) r=r_{0}$ for $\mathrm{NO}, \mathrm{CO}, \mathrm{HCl}$, and $\mathrm{HBr},{ }^{1}$ and the most generally accepted values for the dissociation energies and equilibrium internuclear distances of these diatomic molecules, ${ }^{2}(\partial \mu / \partial r) r=r_{0}$ is found to be proportional to the dissociation energy divided by the square of the equilibrium internuclear distance. A graph of $(\partial \mu / \partial r) r=r_{0}$ as a function of $E / r_{0}{ }^{2}$ is given in Fig. 1. The values used for $E, r_{0}$, and $(\partial \mu / \partial r) r=r_{0}$ are given in Table I. It is pleasant to observe

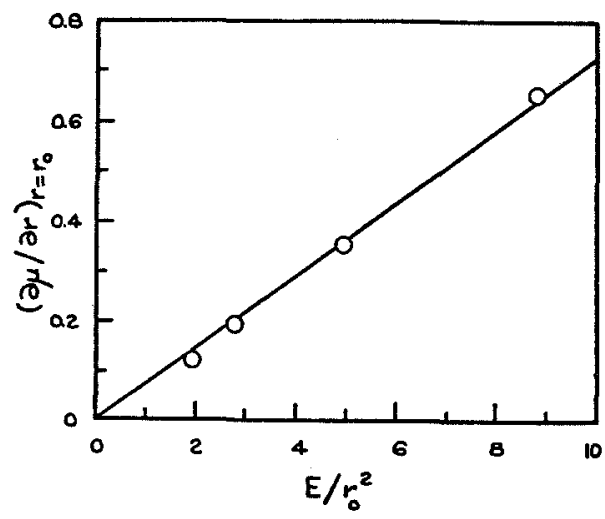

FIG. 1. Graph of $(\partial \mu / \partial r) r=r_{0}$ in $e$ (electronic charge units) as a function of $E / r_{0}^{2}\left(e v / A^{2}\right)$.
TABLE I. Molecular parameters.

\begin{tabular}{cccc}
\hline & $\left(\frac{\partial u}{\partial r}\right)_{r=r_{0}}$ & $r_{0}(\mathrm{~A})$ & $E(\mathrm{ev})$ \\
\hline $\mathrm{HBr}$ & $0.121 e$ & 1.414 & 3.75 \\
$\mathrm{HCl}$ & $0.198 e$ & 1.275 & 4.43 \\
$\mathrm{NO}$ & $0.352 e$ & 1.151 & 6.48 \\
$\mathrm{CO}$ & $0.655 e$ & 1.128 & 11.11 \\
\hline
\end{tabular}

that the data may be correlated so that a straight line goes through the origin, which, in a sense, constitutes a fifth plotted point.

The higher values of $\mathrm{CO}$ and NO, which are perhaps more widely accepted, were used here. It is interesting that the value of $(\partial \mu / \partial r)_{r=r_{0}}$ reported by Penner and Weber for NO, which differs appreciably from previously reported values, correlates with these results. It is further interesting to note that the constant of proportionality has a value of approximately one electronic charge per cubic angstrom.

A fair correlation for overtone intensities on $\mathrm{HBr}, \mathrm{HCl}$, and $\mathrm{CO}$ has also been noted by plotting one of the possible values of $\left(\partial^{2} \mu / \partial r^{2}\right) r=r_{0}$ against $E / r_{0}{ }^{3}{ }^{3}$ These relations should be useful for estimating infrared intensities of molecules such as $\mathrm{HF}$ and $\mathrm{OH}$, where measurement is difficult.

* Supported by the U. S. Office of Naval Research, Contract Nonr$220(03)$, NR 015210.

1S. S. Penner and D. Weber, J. Chem. Phys. 21, 649 (1953); 19, 135 (195i)

$2 \mathrm{G}$. Herzberg, Spectra of Diatomic Molecules (D. Van Nostrand Company Inc., New York, 1950); A. Gaydon, Dissociation Energies and Spectra of Diatomic Molecules (John Wiley and Sons, Inc., New York, 1947).

3In the paper of S. S. Penner and D. Weber, J. Chem. Phys. 21, 649 (1953), the coefficients of $\xi^{2}$ in Eq. (4) for NO should be changed to 8.77 $\times 10^{-18}$ or $-3.06 \times 10^{-18}$; in $\mathrm{Eq}$. (5) for $\mathrm{HCl}$ to $2.96 \times 10^{-18}$ or -0.140 $\times 10^{-18}$. The indicated corrections are the result of recalculation of appropriate coefficients using (a) the intensity data given in the original paper and (b), other required spectroscopic constants listed by Herzberg, reference 2. 